\title{
Lambda-Restricted Crystal-Storing Histiocytosis of Stomach: A Case Report and Review of Literature
}

\author{
Nalini Bansal ${ }^{1}$, Pankaj Puri ${ }^{2}$, Nishant Nagpal ${ }^{2}$, Rahul Naithani ${ }^{3}$, Rahul Gupta ${ }^{4}$ \\ 1. Histopathology, Fortis Escorts Heart Institute, New Delhi, IND 2. Gastroenterology and Hepatology, Fortis Escorts \\ Heart Institute, New Delhi, IND 3. Hematology and Oncology, Max Hospital Saket, New Delhi, IND 4. Gastrointestinal \\ Surgery, Synergy Institute of Medical Sciences, Dehradun, IND
}

Corresponding author: Nalini Bansal, drnalinibansal@yahoo.com

\begin{abstract}
Crystal-storing histiocytosis (CSH) is a rare tissue phenomenon that is usually associated with lymphoproliferative diseases. The disease is characterized by prominent collections of macrophages with abundant eosinophilic cytoplasm and fibrillary cytoplasmic inclusions. The inclusions appear as linear crystals within the macrophages which are usually kappa restricted. The disease usually involves lungs, lymph nodes, bone marrow, thymus and spleen with rare involvement of the gastrointestinal tract. We report a rare case of lambda-restricted CSH of the stomach. The diagnosis of CSH triggered further hematological evaluation. The patient was later diagnosed to have diffuse large B-cell lymphoma involving lymph nodes and bone marrow. He received chemotherapy for the same and is on regular follow up. The index case highlights the need to identify $\mathrm{CSH}$ of stomach prompting evaluation for hematological malignancies and to increase its awareness among clinicians and pathologists.
\end{abstract}

Categories: Internal Medicine, Pathology, Gastroenterology

Keywords: histiocytosis, stomach ulcers, non-hodgkins lymphoma, linear crystals, monoclonal immunoglobulins

\section{Introduction}

Crystal-storing histiocytosis (CSH) is a rare disorder of histiocytic proliferation that usually occurs in patients with underlying hematological malignancies. Histiocytic proliferation shows a unique accumulation of crystals of monoclonal immunoglobulins. The knowledge of this entity is crucial to avoid misdiagnosis of plasma cell neoplasms as immunoglobulin deposition is a usual feature of the latter. The disease usually involves the lung, lymph node, bone marrow, thymus and spleen with rare involvement of the gastrointestinal tract. Involvement of the stomach is rarely reported in the English literature [1-9]. We report the case of lambda-restricted gastric $\mathrm{CSH}$, which later uncovered an underlying diffuse large B-cell lymphoma (DLBCL) involving multiple lymph nodes and bone marrow.

Review began 05/07/2021 Review ended 05/12/2021 Published 05/13/2021

\section{(c) Copyright 2021}

Bansal et al. This is an open access article distributed under the terms of the Creative Commons Attribution License CC-BY 4.0., which permits unrestricted use, distribution, and reproduction in any medium, provided the original author and source are credited.

\section{Case Presentation}

An 86-year-old male presented with complaints of two episodes of hematemesis and abdominal pain for one day. On clinical examination, the patient was hemodynamically stable with no jaundice, clubbing, cyanosis or edema. Abdominal examination revealed mild tenderness in the epigastric region. There were no neurological deficits. Laboratory investigations revealed hemoglobin $12.0 \mathrm{~g} / \mathrm{dL}$ (normal range: 13.0-17.0 $\mathrm{g} / \mathrm{dL})$, white blood cell count 8.18 thou/uL (4.0-10.0 thou/uL), platelet count 174 (150-410 thou/uL), hematocrit 35.5 (40\%- 50\%) with unremarkable differential count. The coagulation profile, liver and renal function tests were within the normal range. Serum lactate dehydrogenase level was elevated [473 U/L (normal range: 135-225 U/L)].

In view of gastrointestinal bleed, an emergency upper gastrointestinal endoscopy (UGIE) was done that showed Schatzki ring, Forrest IIB gastric ulcer in the antrum along the lesser curvature with adherent clot. Inj. Adrenaline $(1: 10,000)$ was injected in all four quadrants of the ulcer. He was started on intravenous proton pump inhibitors. Repeat UGIE done two days later showed large ulcer seen in antrum at the incisura, clean based (Forrest III) with surrounding induration (Figures $1 a, 1 b$ ). Multiple biopsies were taken. The rapid urease test for Helicobacter pylori was positive. Another small ulcer was seen in the antrum with patchy erythema and induration. 


\section{Cureus}

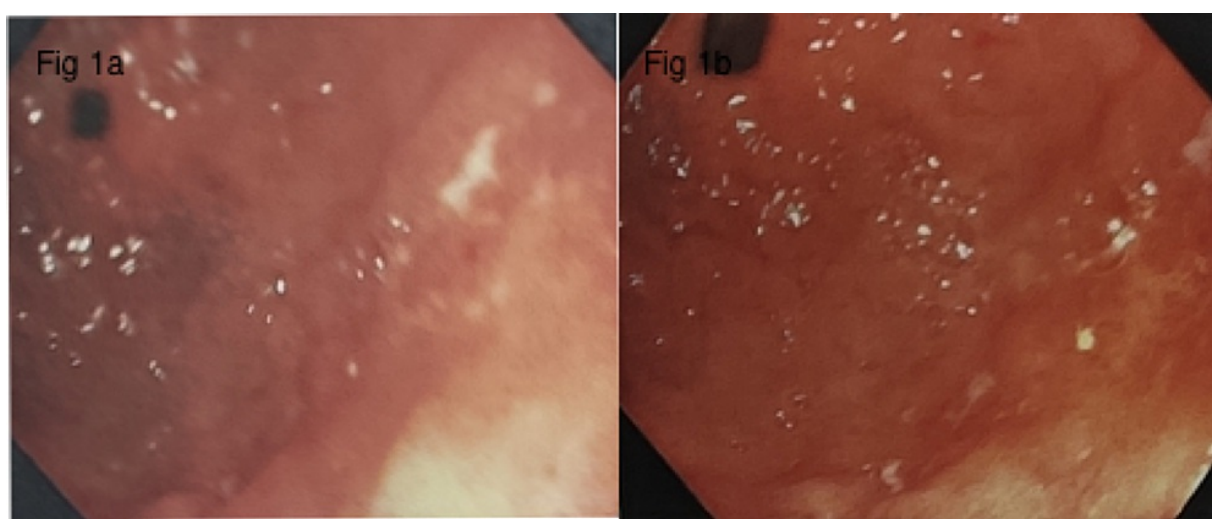

FIGURE 1: Upper gastrointestinal endoscopy at admission (a) and after two days (b) showing the gastric ulcer located in the antrum.

The patient improved symptomatically. He was started on Helicobacter pylori eradication therapy (sequential therapy). The gastric biopsy tissue revealed the presence of large cells with abundant eosinophilic cytoplasm and the presence of linear crystals. Immunohistochemistry (IHC) performed for plasma cells (CD 138) was negative, and the cytoplasmic content was strongly lambda positive with weak kappa staining (Figures $2 a-2 d$ ). The large cells were $\mathrm{CD} 68$ positive and negative for pancytokeratin (PanCK), leukocyte common antigen (LCA) and CD 20. The final diagnosis of CSH was rendered.

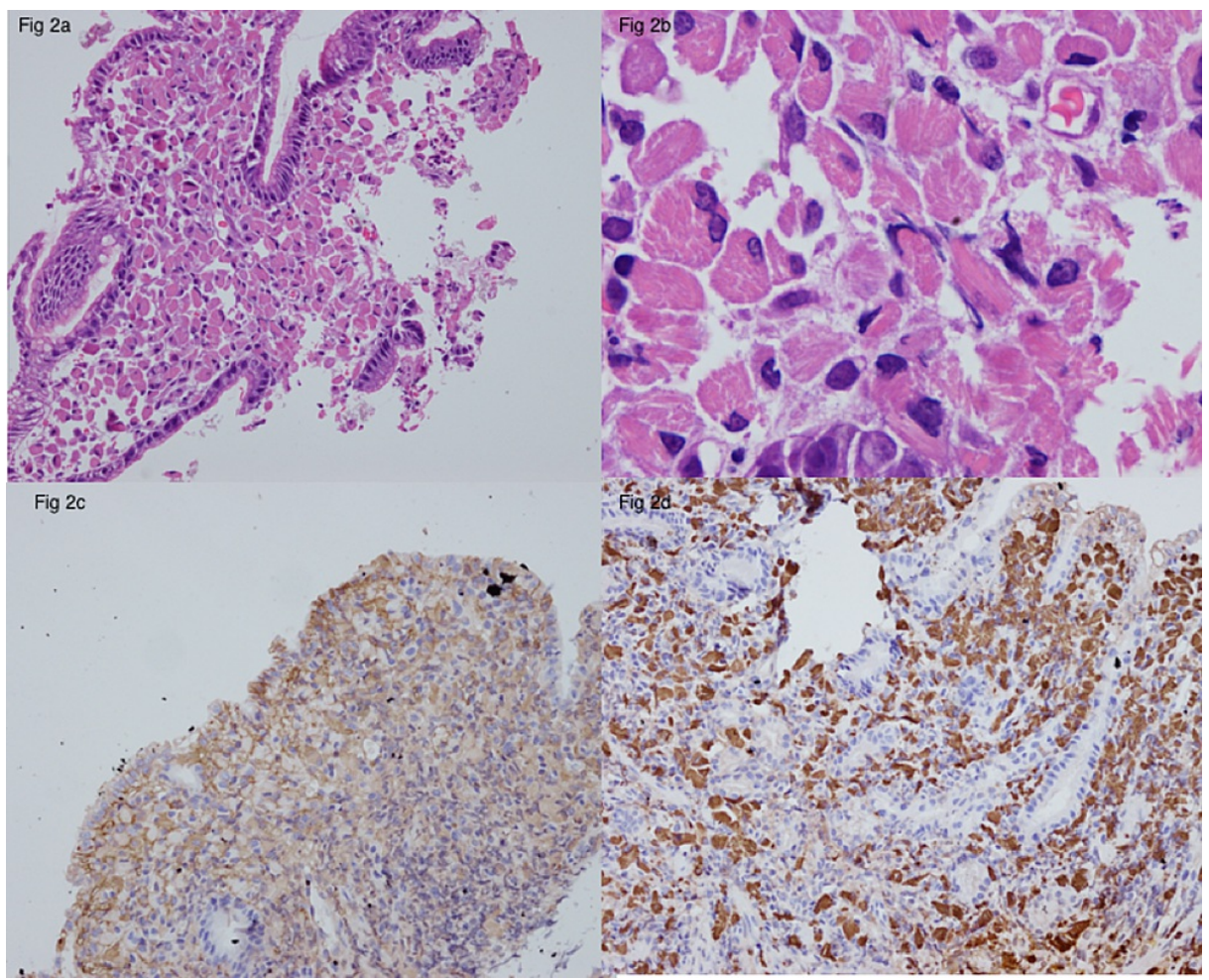

FIGURE 2: Microscopic examination of the gastric biopsy showing histiocyte aggregates in lamina propria (a) and crystals within histiocytes (b). Immunohistochemistry showing kappa (c) and lambda (d) within the histiocytes.

In view of $\mathrm{CSH}$, the patient underwent further evaluation for the myeloma panel which was negative. The patient underwent a PET scan, which showed fluorodeoxyglucose (FDG) avid lesions in multiple lymph nodes and spleen. He later underwent trucut biopsy from the left cervical lymph node. The biopsy tissue showed features of DLBCL. In view of lymphoma, he also underwent bone marrow aspiration and biopsy, which showed marrow infiltration by B-cell Non-Hodgkin's lymphoma. As the performance status of the 
patient was good, he was started on prednisolone keeping his age in mind. He tolerated the chemotherapy well and is now on regular follow up for one year.

\section{Discussion}

$\mathrm{CSH}$ is a rare disease composed of histiocytes with an abnormal intra-lysosomal accumulation of immunoglobulin as crystals [1]. The largest review of CSH involving 80 patients by Dogan et al. found an association with lymphoproliferative plasma cell disorder in $90 \%$ cases [10]. The associated lymphomas were B-lymphoproliferative disorders comprising marginal zone lymphoma with plasmacytic differentiation, lymphoplasmacytic lymphoma, monoclonal gammopathy of uncertain significance and plasma cell neoplasms. Most cases of CSH were identified in the head and neck (35\%), followed by lung and pleura, bone marrow, kidney, lymph nodes, skin and others. The most common site of CSH in the head and neck region was the eye/orbit [10].

The median age of the patients is 60 years (range: $17-81$ years) [1-9]. The male to female ratio is nearly 1:1. The disease can occur in a localized or generalized form. Localized disease is confined to a single organ or site seen in nearly $58 \%$ cases compared to generalized form, which involves around $42 \%$ cases [10]. The clinical features depend on the site of involvement. Histology in most cases shows the presence of histiocytes containing eosinophilic inclusions within the cytoplasm. The eosinophilic inclusions within the cytoplasm appear as non-refractile, nonpolarizable linear crystals [1]. The inclusions are mainly phagocytized excessive light chains that accumulate within the lysosomes of histiocytes. The light chains can be polyclonal or monoclonal. Monoclonal light chains are typically kappa isotype and rarely of lambda isotype. In the present case, the accumulated light chains showed lambda restriction.

The histiocytes in CSH need to be differentiated from Russel body gastritis. In Russel body gastritis, the cells accumulating the immunoglobulin are plasma cells and not histiocytes. Another feature that differentiates the two disorders are the nature of immunoglobulin deposits, which appear as globular deposits within the cell in Russel body gastritis as opposed to linear crystals in CSH [11]. There are few reported cases of linear crystal deposits within histiocytes other than immunoglobulins such as cystine deposits, Charlot Layden crystals and clofazimine-induced crystals [12-14]. The identification of CSH prompts further evaluation for hematological disorders. Treatment and prognosis are varied according to the underlying disease.

Though CSH has been reported from various sites within the body, involvement of the stomach is very rare. The clinicopathological features of the reported cases have been presented in Table 1 [1-9]. CSH localized to the stomach was seen in $85 \%$ cases (11/13 cases). Cases of gastric CSH has been associated with lymphoproliferative disorders in 66\% cases inclusive of monoclonal B-cell lymphoproliferative disorders (multiple myeloma, lymphoplasmacytic lymphoma, extranodal marginal zone lymphoma and mantle cell lymphoma). DLBCL was found to be associated in only one case report as was seen in our case.

Monoclonality was identified in $66 \%$ cases on IHC for kappa and lambda light chains with $87 \%$ cases showing kappa restriction. Lambda restriction was noted in only one case. In the index case, the histiocytes showed lambda restriction. Cases of CSH included are only those cases where histiocytes show crystal accumulation. Cases where plasma cells showed similar crystal were disregarded in the study as they can now be better designated as Russell body gastritis.

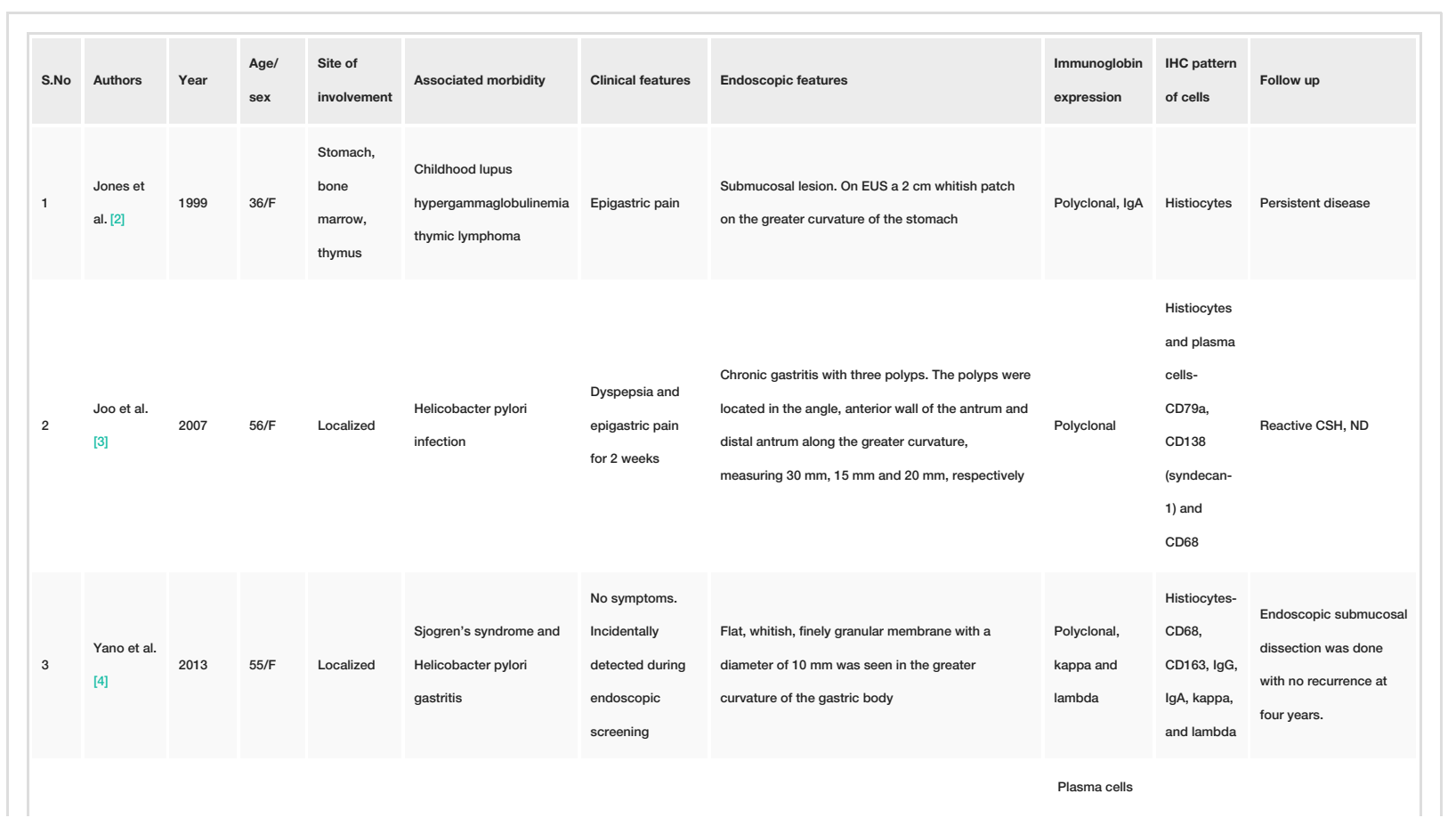




\section{Cureus}

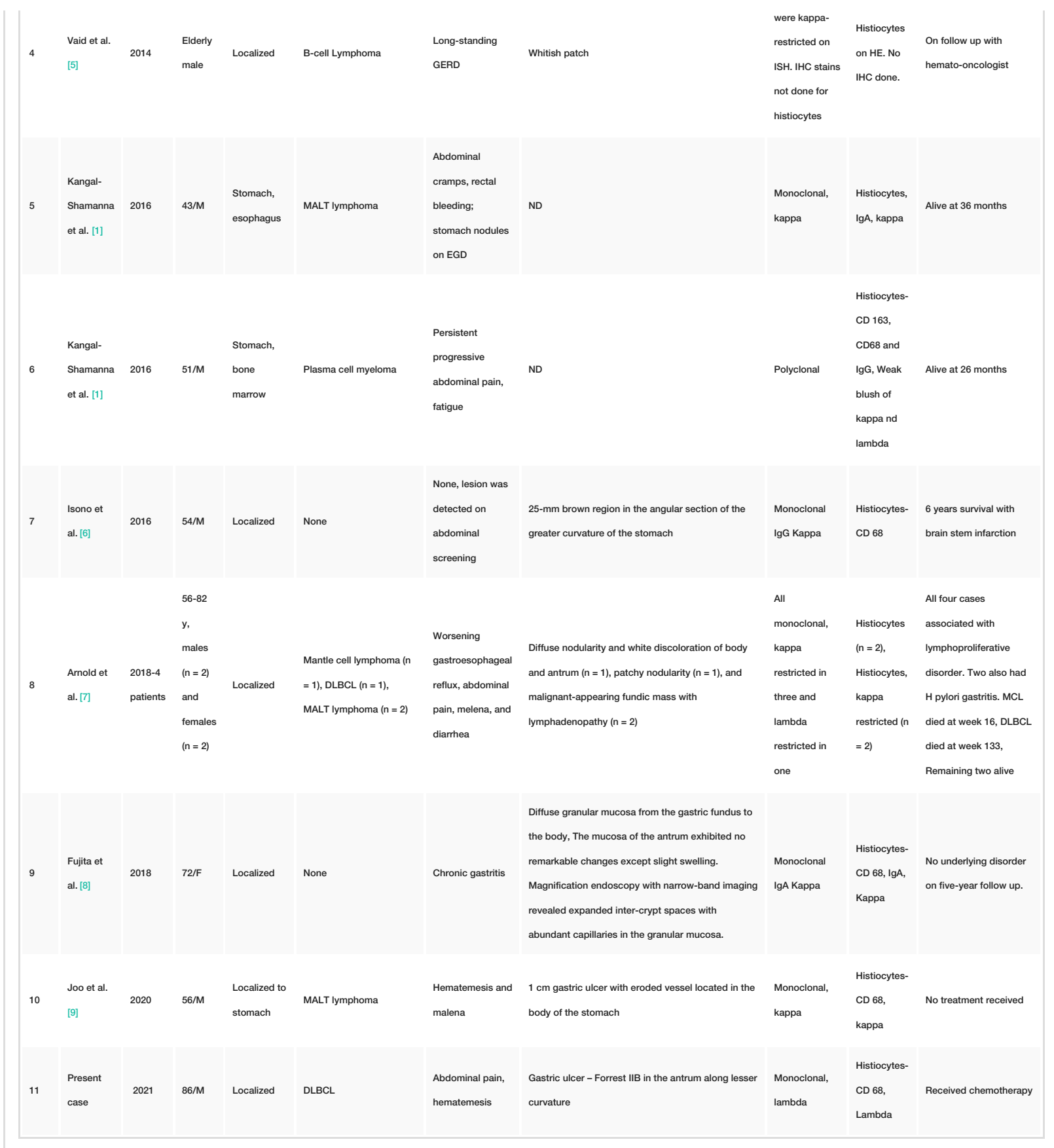

TABLE 1: A brief literature review of reported cases of crystal-storing histiocytosis of the stomach.

CSH - crystal-storing histiocytes, DLBCL - diffuse large B-cell lymphoma, EUS - endoscopic ultrasound, F - female, GERD - gastroesophageal reflux disease, $\mathrm{HE}$ - hematoxylin and eosin, IHC - immunohistochemistry, M - male, MALT - mucosa-associated lymphoid tissue, MCL - mantle cell lymphoma, ND - not described

\section{Conclusions}

Gastric CSH is a very rare pathological finding. It should prompt further hematological work up as most of the cases are associated with lymphoproliferative disorders. High clinical suspicion and increased awareness among clinicians and pathologists are required to avoid delay in the diagnosis and treatment of underlying hematological disorders.

\section{Additional Information}

\section{Disclosures}


Human subjects: Consent was obtained or waived by all participants in this study. Conflicts of interest: In compliance with the ICMJE uniform disclosure form, all authors declare the following: Payment/services info: All authors have declared that no financial support was received from any organization for the submitted work. Financial relationships: All authors have declared that they have no financial relationships at present or within the previous three years with any organizations that might have an interest in the submitted work. Other relationships: All authors have declared that there are no other relationships or activities that could appear to have influenced the submitted work.

\section{References}

1. Kanagal-Shamanna R, Xu-Monette ZY, Miranda RN, et al.: Crystal-storing histiocytosis: a clinicopathological study of 13 cases. Histopathology. 2016, 68:482-91. 10.1111/his.12768

2. Jones D, Bhatia VK, Krausz T, Pinkus GS: Crystal-storing histiocytosis: a disorder occurring in plasmacytic tumors expressing immunoglobulin kappa light chain. Hum Pathol. 1999, 30:1441-8. 10.1016/s00468177(99)90166-1

3. Joo M, Kwak JE, Chang SH, Kim H, Chi JG, Moon YS, Kim KM: Localized gastric crystal-storing histiocytosis. Histopathology. 2007, 51:116-9. 10.1111/j.1365-2559.2007.02710.x

4. Yano Y, Nagahama T, Matsui T, et al.: Gastric crystal-storing histiocytosis detected with asymptomatic Sjögren's syndrome: report of a case and summary. Clin J Gastroenterol. 2013, 6:237-42. 10.1007/s12328013-0388-8

5. Vaid A, Caradine KD, Lai KK, Rego R: Isolated gastric crystal-storing histiocytosis: a rare marker of occult lymphoproliferative disorders. J Clin Pathol. 2014, 67:740-1. 10.1136/jclinpath-2014-202247

6. Isono Y, Baba Y, Tanaka H, et al.: A case of gastric crystal-storing histiocytosis . Nihon Shokakibyo Gakkai Zasshi. 2016, 113:968-74. 10.11405/nisshoshi.113.968

7. Arnold CA, Frankel WL, Guo L, et al.: Crystal-storing histiocytosis in the stomach: a Clue to Subtle Hematolymphoid Malignancies. Am J Surg Pathol. 2018, 42:1317-24. 10.1097/PAS.0000000000001097

8. Fujita Y, Oikawa H, Toya Y, et al.: Gastric crystal-storing histiocytosis without any underlying disorders: report of a case. Hum Pathol Case Rep. 2018, 14:73-7. 10.1016/j.ehpc.2018.09.003

9. Joo M, Kim NH: Gastric crystal-storing histiocytosis with concomitant mucosa-associated lymphoid tissue lymphoma. J Pathol Transl Med. 2020, 54:332-5. 10.4132/jptm.2020.04.20

10. Dogan S, Barnes L, Cruz-Vetrano WP: Crystal-storing histiocytosis: report of a case, review of the literature (80 cases) and a proposed classification. Head Neck Pathol. 2012, 6:111-20. 10.1007/s12105-011-0326-3

11. Erbersdobler A, Petri S, Lock G: Russell body gastritis: an unusual, tumor-like lesion of the gastric mucosa . Arch Pathol Lab Med. 2004, 128:915-7. 10.1043/1543-2165(2004)128<915:RBGAUT>2.0.CO;2

12. Gebrail F, Knapp M, Perrotta G, Cualing H: Crystalline histiocytosis in hereditary cystinosis . Arch Pathol Lab Med. 2002, 126:1135. 10.1043/0003-9985(2002)126<1135:CHIHC>2.0.CO;2

13. Lewis JT, Candelora JN, Hogan RB, Briggs FR, Abraham SC: Crystal-storing histiocytosis due to massive accumulation of charcot-leyden crystals: a unique association producing colonic polyposis in a 78-year-old woman with eosinophilic colitis. Am J Surg Pathol. 2007, 31:481-5. 10.1097/01.pas.0000213420.46127.9c

14. Sukpanichnant S, Hargrove NS, Kachintorn U, et al.: Clofazimine-induced crystal-storing histiocytosis producing chronic abdominal pain in a leprosy patient. Am J Surg Pathol. 2000, 24:129-35. 10.1097/00000478-200001000-00016 